\title{
Study of optical properties of the $\mathrm{NV}$ and $\mathrm{SiV}$ centres in diamond at high pressures
}

\author{
S. G. Lyapin ${ }^{1}$, I. D. Ilichev ${ }^{1}$, A. P. Novikov ${ }^{1}$, V. A. Davydov ${ }^{1}$, V. N. Agafonov ${ }^{2}$ \\ ${ }^{1}$ L. F. Vereshchagin Institute for High Pressure Physics, Russian Academy of Sciences, Troitsk, Moscow, Russia \\ ${ }^{2}$ L. E. M. A., UMR CNRS-CEA 6157, University F. Rabelais, Tours, France \\ vdavydov@hppi.troitsk.ru
}

PACS 33.50.Dq, 62.50-p

DOI 10.17586/2220-8054-2018-9-1-55-57

\begin{abstract}
We report photoluminescence studies of micro- and nano-sized diamonds with $\mathrm{NV}^{0}, \mathrm{NV}^{-}$and $\mathrm{SiV}^{-}$centers under hydrostatic pressure up to $50 \mathrm{GPa}$. Diamonds have been obtained by high-pressure high-temperature (HPHT) treatment of metal-free growth systems based on mixtures of hydrocarbon, fluorocarbon, and silicone-containing organic compounds.
\end{abstract}

Keywords: diamond, colour centres, high pressures, DAC.

Received: 20 June 2017

Revised: 11 October 2017

\section{Introduction}

Color centers in diamond are considered now as an important basic element for different quantum-physical and biomedical applications. Although the properties of color centers in diamond have been studied very intensively for a long time, the pressure effect on photoluminescence is not well known. Knowledge of changes in the energy of the zero-phonon line (ZPL) with hydrostatic stress is crucial in analyzing temperature dependences [1,2], isotope shift [3] of ZPL. To our knowledge, the pressure coefficient was measured only for $\mathrm{NV}^{-}$centers [4,5].

Here, we report pressure effect on zero-phonon line in $\mathrm{SiV}^{-}, \mathrm{NV}^{0}$ and $\mathrm{NV}^{-}$centers under hydrostatic pressure up to $50 \mathrm{GPa}$.

\section{Experimental}

Homogeneous mixtures of naphthalene $\left(\mathrm{C}_{10} \mathrm{H}_{8}\right)$, fluorographite $\left(\mathrm{CF}_{1.1}\right)$ and tetrakis(trimethylsilyl)silane $\left(\mathrm{C}_{12} \mathrm{H}_{36} \mathrm{Si}_{5}\right)$ were used as starting materials for synthesis of diamond with optically active $\mathrm{SiV}^{-}, \mathrm{NV}^{0}$ and $\mathrm{NV}^{-}$point defects. The synthesis was performed in a high-pressure apparatus of "Toroid" type [6]. Cylindrical samples of the initial mixtures ( $4 \mathrm{~mm}$ diameter and $5 \mathrm{~mm}$ height) obtained by cold pressing were put into graphite containers which were placed in the high-pressure apparatus. The experimental procedure consisted in loading the apparatus up to $8 \mathrm{GPa}$, heating up to the desired temperature $\left(\sim 1200{ }^{\circ} \mathrm{C}\right)$ and short isothermal exposure under constant load for 5-10 s. The SEM images of the obtained products, which are mixtures of nano- and submicrometer-size fraction of diamond, show that the formation of diamond occurs with virtually $100 \%$ yield.

Photoluminescence (PL) spectra were recorded using the $488 \mathrm{~nm} \mathrm{Ar}^{+}$laser line for excitation and a triplegrating spectrometer (Princeton Instruments TriVista 555) with a liquid-nitrogen-cooled CCD detector. Diamond samples, representing distinct agglomerates of micro- and nanoscale diamonds, were placed in a diamond-anvil cell (DAC) along with ruby crystal, serving as a pressure sensor. Helium was used as pressure-transmitting medium. For measurements at room temperature, a $50 \times$ objective $(\mathrm{NA}=0.50)$ of a confocal microscope $($ Olympus $\mathrm{BX} 51)$ was used to focus the laser beam and to collect the PL signal. To perform measurements at $80 \mathrm{~K}$, the DAC was put into a He cryostat (Oxford Instruments OptistatSXM) and an achromatic lens was used for focusing the laser beam and collecting the signal. The laser spot on the sample inside the cryostat was $\sim 5 \mu \mathrm{m}$.

\section{Results and discussion}

Figure 1 shows typical photoluminescence spectra collected on diamonds doped with silicon of natural isotope composition. Because of unintentional doping of diamond with nitrogen occurred during synthesis, some of our samples contain nitrogen-vacancy defects (neutral $\mathrm{NV}^{0}$ and negatively charged $\mathrm{NV}^{-}$) detectable already at room temperature $296 \mathrm{~K}$ [6]. Two types of diamonds were used in pressure experiments i) with high concentration $\mathrm{SiV}^{-}$ centers in order to track $\mathrm{ZPL}$ from $\mathrm{SiV}^{-}$centers; ii) with low concentration $\mathrm{SiV}^{-}$centers to allow detection of ZPL from NV centers. We were able to follow ZPL from $\mathrm{SiV}^{-}$and $\mathrm{NV}^{0}$ centers up to $\sim 52 \mathrm{GPa}$ (the maximum pressure achieved in our experiment). However, ZPL from $\mathrm{NV}^{0}$ centers became undetectable above $\sim 40 \mathrm{GPa}$. 


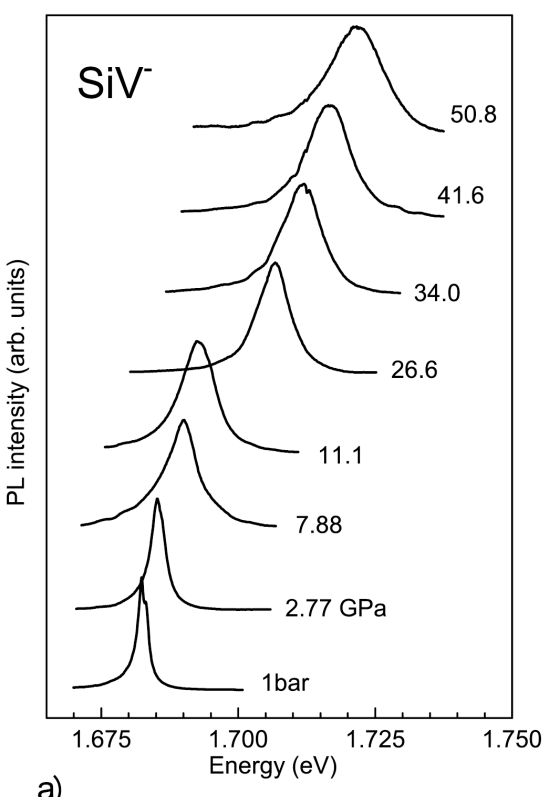

a)

FIG. 1. Normalized PL spectra of diamond with $\mathrm{SiV}^{-}$ pressures at $80 \mathrm{~K}$. The background for spectra in panel

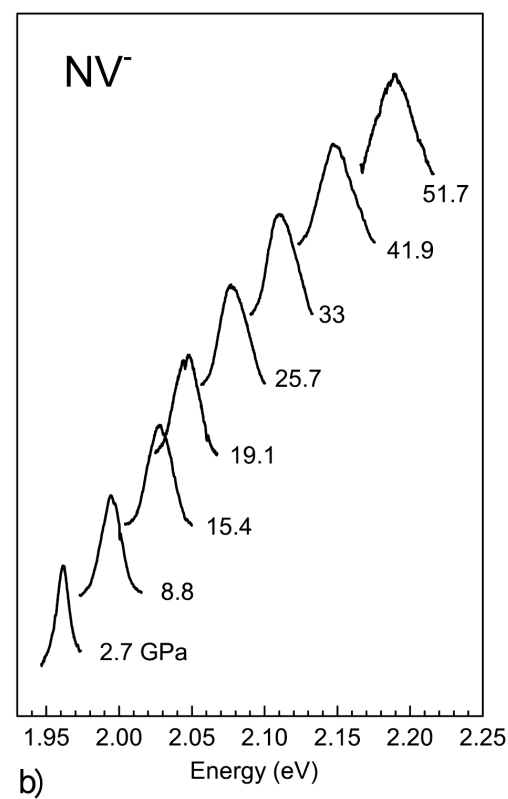

(a), $\mathrm{NV}^{-}$(b), $\mathrm{NV}^{0}$ (c) centres at various

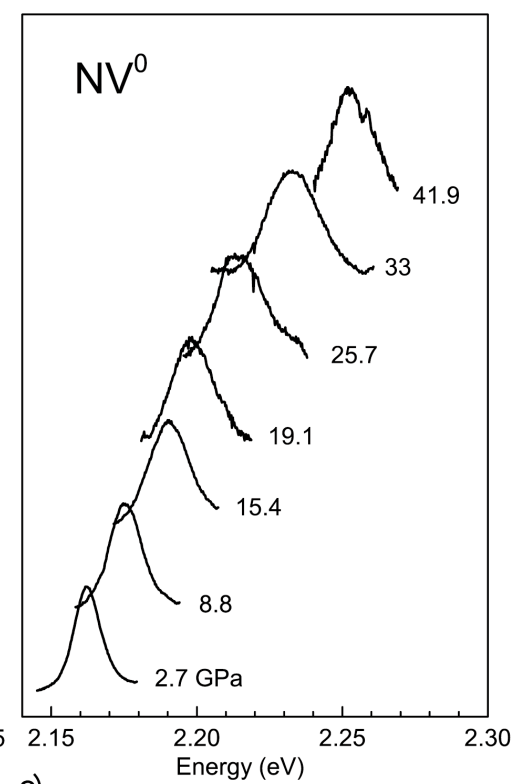

c) (b) and (c) was subtracted for clarity

Pressure dependences of ZPL of negatively charged $\mathrm{NV}^{-}$and $\mathrm{SiV}^{-}$centers at 80 and $296 \mathrm{~K}$ were approximated by the second order polynomial equation: $E(P)=E_{0}+\alpha P+\beta P^{2}$, while that of the neutral $\mathrm{NV}^{0}$ center was found to be linear with rather good precision (Fig. 2, Table 1). If we use equation of state for diamond [7] to replot ZPL position versus natural logarithm of lattice constant of diamond $\left(\ln \left[a(P) / a_{0}\right]\right)$, the nonlinear dependence of peak position for negatively charged $\mathrm{NV}$ and $\mathrm{SiV}$ centers remains.
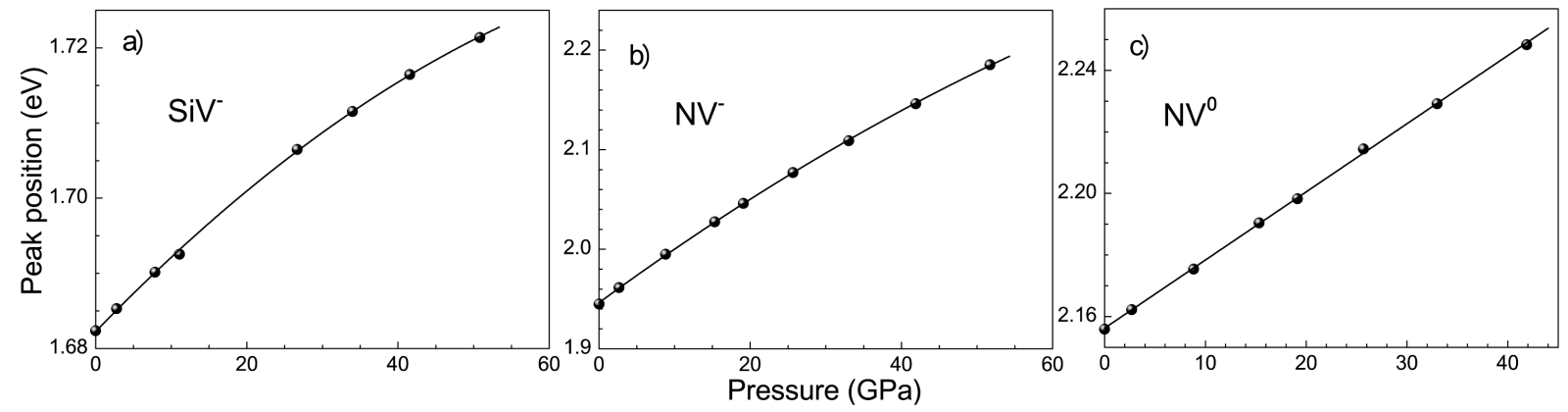

FIG. 2. Pressure dependence of $\mathrm{ZPL}$ in $\mathrm{SiV}^{-}$(a), $\mathrm{NV}^{-}$(b), $\mathrm{NV}^{0}$ (c) centres at $80 \mathrm{~K}$

The pressure coefficients $\alpha$ for $\mathrm{NV}^{-}$centers are in good agreement with those obtained at $77 \mathrm{~K}$ [4] and at room temperature [5] and they are reasonably close to the pressure coefficients of the direct and indirect gaps in diamond $[8,9]$. The obtained data show that the effect of pressure on the position of zero-phonon lines most sharply manifested in the case $\mathrm{NV}^{-}$centers, for which the $\alpha$ coefficient value at $296 \mathrm{~K}$ is equal to $5.81 \mathrm{meV} / \mathrm{GPa}$. In the case of the $\mathrm{SiV}^{-}$center, the $\alpha$ coefficient is equal to $1.09 \mathrm{meV} / \mathrm{GPa}$, which is 5 -fold less than the previous value. The $\alpha$ coefficient value for neutral NV centers is $2.14 \mathrm{meV} / \mathrm{GPa}$, meaning it has an intermediate value between the corresponding values of negatively charged $\mathrm{NV}^{-}$and $\mathrm{SiV}^{-}$centers. Significant difference in the $\alpha$ coefficient values of negatively charged $\mathrm{NV}^{-}$and $\mathrm{SiV}^{-}$centers is associated, obviously, with the different structures for these two types of diamond lattice defects. 
TABLE 1. Pressure coefficients for $\mathrm{ZPL}$ in $\mathrm{SiV}^{-}, \mathrm{NV}^{-}$and $\mathrm{NV}^{0}$ centres at 296 and $80 \mathrm{~K}$

\begin{tabular}{|c|c|c|c|c|c|c|}
\hline \multicolumn{7}{|c|}{$E(P)=E_{\mathbf{0}}+\alpha \cdot P+\beta \cdot P^{2}$} \\
\hline & \multicolumn{3}{|c|}{$296 \mathrm{~K}$} & \multicolumn{3}{c|}{$80 \mathrm{~K}$} \\
\hline Center & $\begin{array}{c}E_{0} \\
(\mathrm{eV})\end{array}$ & $\begin{array}{c}\alpha \\
(\mathrm{meV} / \mathrm{GPa})\end{array}$ & $\begin{array}{c}\beta \cdot 10^{-3} \\
\left(\mathrm{meV} / \mathrm{GPa}^{2}\right)\end{array}$ & $\begin{array}{c}E_{0} \\
(\mathrm{eV})\end{array}$ & $\begin{array}{c}\alpha \\
(\mathrm{meV} / \mathrm{GPa})\end{array}$ & $\begin{array}{c}\beta \cdot 10^{-3} \\
\left(\mathrm{meV} / \mathrm{GPa}^{2}\right)\end{array}$ \\
\hline $\mathrm{SiV}^{-}$ & 1.680 & 1.09 & -5.7 & 1.682 & 1.04 & -5.3 \\
\hline $\mathrm{NV}^{-}$ & 1.943 & 5.81 & -25 & 1.946 & 5.57 & -19 \\
\hline $\mathrm{NV}^{0}$ & 2.156 & 2.14 & 0 & 2.156 & 2.21 & 0 \\
\hline
\end{tabular}

\section{Acknowledgements}

The work was supported by the Russian Foundation for Basic Research (Grant No. 18-03-00936).

\section{References}

[1] Neu E., Hepp C., et al. Low-temperature investigations of single silicon vacancy colour centres in diamond. New J. Physics, 2013, 15 (4), 043005 .

[2] Jahnke K.D., Sipahigil A., et al. Electronphonon processes of the siliconvacancy centre in diamond. New J. Physics, 2015 , $17,043011$.

[3] Collins A.T., Davies G., Kanda H., Woods G.S. Spectroscopic Studies of C-13 Synthetic Diamond. J. Physics C: Solid State Physics, 1988, 21 (8), P. 1363-1376.

[4] Kobayashi M., Nisida Y. High-Pressure Effects on Photoluminescence Spectra of Color-Centers in Diamond. Jpn. J. Appl. Phys., 1993, 32, P. 279-281.

[5] Doherty M.W., Struzhkin V.V., et al. Electronic properties and metrology applications of the diamond $\mathrm{NV}^{-}$center under pressure. Phys. Rev. Lett., 2014, 112 (4), 047601.

[6] Davydov V.A., Rakhmanina A.V., et al. Production of nano- and microdiamonds with Si-V and N-V luminescent centers at high pressures in systems based on mixtures of hydrocarbon and fluorocarbon compounds. JETP Lett., 2014,99 (10), P. $585-589$.

[7] Occelli F., Loubeyre P., Letoullec R. Properties of diamond under hydrostatic pressures up to 140 GPa. Nature Mater, 2003, 2 (3), P. 151-154.

[8] Wei S.H., Zunger A. Predicted band-gap pressure coefficients of all diamond and zinc-blende semiconductors: Chemical trends. Phys. Rev. B, 1999, 60 (8), P. 5404-5411.

[9] Onodera A., Hasegawa M., et al. Pressure-Dependence of the Optical-Absorption Edge of Diamond. Phys. Rev. B, 1991, 44 (22), P. 12176-12179. 